\title{
Estrategias de apoyo: requerimientos del estudiantado con discapacidad visual
}

\author{
Martha Lilia Gross Martínez \\ Maestra en Psicopedagogía \\ Docente e investigadora \\ Escuela de Orientación y Educación Especial, Facultad de Educación, Universidad de Costa Rica, Costa Rica \\ Email: martha.gross@ucr.ac.cr \\ Recepción: 30-09-2017 / Aceptación: 15-12-2018
}

\section{Resumen}

El presente artículo de revisión bibliográfica explora una serie de estrategias de apoyo que orientan de manera particular al profesorado, sobre diferentes opciones para adecuar la propuesta curricular, acorde a las necesidades educativas de la población estudiantil con discapacidad visual. El proceso metodológico utilizado se fundamentó en una revisión descriptiva, mediante el trabajo en etapas para la búsqueda, organización, selección y análisis de la información pertinente a la temática de interés.

Desde este punto de vista, se plantean propuestas teóricas sobre la relación discapacidad visual y cognición, así como diversos criterios para llevar a cabo el ajuste del plan educativo y dar respuesta efectiva a la diversidad de requerimientos del estudiantado, en el acceso al proceso de enseñanza aprendizaje; en condiciones de equidad. De igual forma, se destaca como aporte significativo la descripción de prácticas relacionadas con las pautas para la asignación de apoyos en el proceso de evaluación, como un componente clave del ejercicio del derecho a una educación de calidad.

Palabras clave: acceso a la educación, discapacidad visual, cognición, estrategias de apoyo, evaluación.

\begin{abstract}
This bibliographic revision article explores a series of supporting strategies that guide teachers in a particular way about different options to adequate the curricular proposal according to the educative needs of the student population with visual disability. The used methodologic process was based on a descriptive revision through the work in stages for the search, organization, selection and analysis of the pertinent information of the topic of interest.

From this point of view, some theoretic proposals are suggested based on the visual and cognitive disability as well as various criteria to carry out the adjustment of the educational plan and to give an effective response to the diversity of the student's requirements, in the access to the teaching-learning process; in conditions of equity. Similarly, the description of practices related to the guidelines for the allocation of supports in the evaluation process stands as a significant contribution, as a key component of the exercise of the right to a quality education.
\end{abstract}

Key Words: Education access, visual disability, cognition, support strategies, evaluation. 


\section{Introducción}

La participación de la población estudiantil con discapacidad visual al sistema de educación general ha promovido, en las últimas décadas, la implementación de una serie de transformaciones a nivel de legislación en los diferentes sectores sociales; y de acciones estratégicas encaminadas hacia procesos más amplios a una sociedad inclusiva. Evidencia de ello ha sido la promulgación de leyes nacionales y convenios internacionales que fortalecen el derecho y el acceso a la educación, entre las cuales se pueden mencionar, para el contexto costarricense, la Ley 7600 de Igualdad de Oportunidades para las Personas con Discapacidad (1996) y la Ley 8661 Convención sobre los Derechos de las Personas con Discapacidad (2008). Así, Tauber (2011), señala que el impacto de la normativa en materia de discapacidad, se visualiza en prácticas sociales, que permiten el fortalecimiento de una sociedad que reconoce como principal riqueza la diversidad humana y el abordaje de los desafíos que ello implica.

Cabe destacar que, esos cambios sociales han incidido en la evolución conceptual y pragmática del ámbito educativo, facilitando el paso de un sistema integracionista a uno fundamentado en la educación inclusiva; que se sustenta a partir de un marco de referencia del derecho de las personas con discapacidad, y recibir una enseñanza de calidad que garantice el acceso de los apoyos requeridos para la consecución del proyecto académico, de las personas con discapacidad, en todas las etapas de su vida estudiantil. En ese sentido, de acuerdo con Reed y Curtis (2012), el grupo de estudiantes con discapacidad visual se enfrenta a una diversidad de retos al asumir un proyecto de estudios, relacionados con el acceso al proceso educativo, la eliminación de las barreras del entorno y la provisión de apoyos que garanticen su participación en igualdad de condiciones. Así, el acompañamiento y soporte que se brinda a través de las diferentes instancias y servicios que propician la accesibilidad debe favorecer la participación de la totalidad del estudiantado, que ingresa a los centros escolares en condiciones de equidad.

$\mathrm{Al}$ respecto, en el estudio se exploraron una serie de estrategias que orientan de manera particular al profesorado sobre diferentes opciones para adecuar el proceso evaluativo en coherencia con la propuesta curricular, acorde a las necesidades educativas de la población estudiantil con discapacidad visual. Brown (1980), indica que la planificación de una prueba para medir el rendimiento escolar, constituye uno de los primeros pasos en dicho proceso, por cuanto se tienen que tomar una serie de decisiones relacionadas con la diversidad de aspectos como longitud y formato de los ítems, entre otros.

Por consiguiente, esta temática se considera un aspecto de suma importancia no solo por las características propias del proceso de evaluación, la elaboración de los ítems y contenidos de la propuesta educativa vincu- 
lado con las diferentes áreas académicas; sino también por la capacidad para dar respuesta a los requerimientos de accesibilidad del estudiantado en condición de discapacidad, y así garantizar al profesorado la oportunidad de contar con evidencias precisas del proceso de enseñanza aprendizaje de la población estudiantil en condiciones de equidad.

\section{Discapacidad visual}

La discapacidad visual se considera como una pérdida o disminución de las habilidades visuales de la persona para la realización de asignaciones y tareas que implican una exigencia del sentido de la vista; por ejemplo: la lectura de textos impresos, copia a distancia de la pizarra y desplazamientos. Esta limitación es generada por una deficiencia de tipo orgánica, lo cual a su vez incide no solo en la magnitud de la misma (leve, moderada o severa), sino también en la funcionalidad y competencia para desenvolverse en el entorno. Asimismo, la mayor parte de las actividades que realiza el ser humano implican un componente cualitativo, por lo que la percepción del detalle a nivel visual es pertinente para contextualizar las necesidades de la persona en las diferentes situaciones y exigencias de estas (León, Polanco, Rovezzi, Contreras y Ramos, 2014). De esta forma, la condición de discapacidad visual se entiende como una limitación significativa de la eficiencia del sistema visual, agrupando dos tipos de funcionalidad: la ceguera y la baja visión; cada una categorizada según sea la pérdida visual.
Según estima la Organización Mundial de la Salud (2010), en el mundo hay aproximadamente 314 millones de personas con discapacidad visual, lo cual representa el $4 \%$ de la población mundial con esa condición. En Costa Rica, los datos del Censo Nacional (2011) indican que hay 251954 personas con discapacidad visual. Esta información permite concebir a una población con necesidades sensoriales muy particulares, que demandan servicios y apoyos en diferentes entornos.

Con el fin de homogenizar criterios conceptuales, León et al (2014), retoman una serie de terminologías planteadas en la Convención del Consejo Internacional de Oftalmología, celebrada en Australia en el año 2002, donde se dieron la tarea de definir el concepto renovado de la discapacidad visual. Al respecto, señalan que la ceguera se refiere a la pérdida total de la visión, lo cual incide en la sustitución de las habilidades visuales. Complementando este concepto, Aquino, García e Izquierdo determinan que la ceguera se presenta cuando se “(...) tiene una pérdida total de la visión, o bien, el remanente que posea es tan pequeño que no le permite desarrollar actividades utilizando esta percepción, es decir, el remanente visual que poseen estas personas es mínimo o simplemente no existe" (2012, p. 9).

Por otro lado, la baja visión hace referencia a grados menores de pérdida visual en que las personas puedan ser apoyadas, significativamente, para mejorar su visión mediante el 
uso de ayudas ópticas y sistemas especializados. De esta forma, Aquino et al señalan que la baja visión se presenta cuando

Una persona acusa una percepción visual disminuida o insuficiente, la cual, a pesar de las ayudas ópticas, sigue estando bajo el promedio de una visión normal; es decir, las personas con baja visión poseen el remanente visual o resto de visión que les permitirá utilizar funcionalmente este sentido; muchas de ellas podrán incluso escribir y leer textos impresos, por lo general amplificados, con las ayudas ópticas que sean necesarias en cada caso, como lupas, lentes $\mathrm{u}$ otros instrumentos que le sirvan para magnificar los caracteres e imágenes que desee ver (2012, p. 9).

En esta misma línea temática, la Organización Nacional de Ciegos Españoles (ONCE) citado en Blanco (2010), refiere que la baja visión está relacionada con la funcionalidad de la persona y afirma que ésta se define "a partir del resto visual suficiente para ver la luz, orientarse por ella y emplearla con propósitos funcionales" (p. 92).

Por consiguiente, este grupo de estudiantes requieren de experiencias educativas significativas y flexibles, de tal forma que se propicie su autonomía y desempeño, en la diversidad de prácticas que se llevan a cabo en el aula, haciendo efectivo su derecho a una educación inclusiva.

\section{Discapacidad visual y cognición}

Las investigaciones y estudios sobre el desarrollo y su relación con el proceso educativo han permitido logros significativos tanto en el campo de la psicología evolutiva como en la educación, lo que ha generado aportes valiosos, principalmente, a partir del siglo XIX. Ello permitió reformas en el sector educativo y la creación de principios pedagógicos que innovaron creencias y postulados en la dinámica educativa. En ese sentido, se destacan investigadores como Dewey (1859-1952), Piaget (1896-1980), Gesell (1880-1961) y Vigotsky (1896-1934), los cuales han constituido una referencia clave en el campo de la psicología y de la educación desde diferentes ámbitos, como bien lo afirma López:

Todos estos avances en la psicología evolutiva y en la educación tuvieron una influencia directa sobre la atención prestada a las personas con deficiencias y concretamente con deficiencias visuales, pues, aunque todavía no se aplicaron estos procedimientos a su educación, al menos se empezó a tomar conciencia de la posibilidad de que recibieran educación y se crearon centros de atención y de institucionalización (2004, p. 24).

Sin embargo, no es hasta la segunda mitad del siglo XX que se inicia un interés particular por llevar a cabo estudios relacionados con las necesidades propias de la población con discapacidad visual, siendo de relevancia el aporte de Natalie Barraga, quien a partir de 1964 realiza una serie de estudios con niños 
con una condición de discapacidad visual severa, con el fin de indagar sobre la eficiencia visual y precisión en la realización de tareas visuales. De esta manera, se empieza a evidenciar mayor disposición para la creación de programas de estimulación, entrenamiento visual y elaboración de pruebas para evaluar la funcionalidad visual (López, 2004). Es en las últimas décadas que se ha generado una mayor tendencia a realizar investigaciones, relacionando la deficiencia y procesos de desarrollo cognitivo. A continuación, se mencionan algunos de ellos:

Wyver y Markham (1999), en su estudio sobre pensamiento divergente y la habilidad para encontrar múltiples soluciones a un problema, en niños con discapacidad visual severa, realizaron comparaciones entre niños con discapacidad visual congénita y niños videntes. Los resultados encontrados sugieren que los factores que contribuyen al desarrollo del pensamiento divergente, puede ser más difícil en niños con discapacidad visual severa que en sus pares videntes. Aun cuando indican que se requiere mayor investigación en el tema, señalan que el pensamiento divergente se correlaciona con una variedad de habilidades académicas y no académicas. Además, señalan que estas diferencias en el desarrollo del pensamiento divergente, refleja mayores diferencias o limitaciones en el desarrollo de los niños con discapacidad visual congénita.

En la misma temática sobre procesos cognitivos y discapacidad visual se encuentra el estudio de Gompel, Van Bon y Schreuder (2004), quienes investigaron sobre aspectos del proceso lector en niños con baja visión y niños videntes. Entre los hallazgos señalan que los niños con baja visión presentan más problemas que los niños videntes en el ordenamiento de letras; y que esto puede ser por el peso dado en los procesos de memoria de trabajo. Con esta información los investigadores señalan que los niños con baja visión requieren mayor tiempo para identificar las letras en las palabras. Además, indican que muchos niños con baja visión presentan la condición de nistagmus (movimiento rápido e involuntario de los ojos) la cual puede causarles dificultades en la fijación e interferir en procesos de ordenamiento de las letras para la lectura, por lo que requieren mayor tiempo para la lectura.

Estos aportes constituyen una perspectiva teórica, que influye en la docencia para operacionalizar los contenidos contemplados en el plan educativo a evaluar, acorde a los requerimientos del estudiantado con discapacidad visual.

\section{Desarrollo}

El proceso metodológico utilizado se fundamentó en una revisión documental descriptiva, mediante el trabajo en etapas para la búsqueda, organización, selección y análisis de la información pertinente a la temática de interés; siguiendo las etapas descritas por Guirao-Goris, J.A; Olmedo Salas, A; Ferrer Ferrandis, E. (2008) como una estrategia enriquecedora para sustentar el análisis respectivo, partiendo de la definición de los objetivos de la revisión; consultando bases de datos 
y fuentes documentales, así como una búsqueda en internet utilizando diferentes bases de datos como: Education Research Complete, Academic Search Complete, ResearchGate. Se determinaron los criterios de selección de documentos haciendo uso de los siguientes descriptores: discapacidad visual, cognición, evaluación, adecuaciones, educación inclusiva y estrategias de apoyos educativos. Posteriormente se organizó la información recolectada, la cual se clasificó por unidades conceptuales acorde a la temática de interés.

\section{Educación Inclusiva}

En el devenir de investigaciones relacionadas con la educación inclusiva se establecen varios aspectos fundamentales: el respeto a la dignidad de las personas, no solo a nivel físico sino mental, la transformación del entorno educativo, los apoyos en el aula, la eliminación de las barreras del aprendizaje y la participación en pleno de la comunidad escolar. Esto conlleva considerar de manera relevante, la acción interdisciplinar y docente vinculada con el tema del derecho a una educación de calidad, en condiciones de equidad e igualdad de oportunidades. En este sentido, la reflexión y la toma de posición de resguardo ante aquellas personas, que presentan condiciones que las ubican en desventaja en los diferentes contextos, es prioritario en el devenir docente. $\mathrm{Al}$ respecto, Parra expresa que "La educación inclusiva constituye un enfoque educativo basado en la valoración de la diversidad como elemento enriquecedor del proceso de enseñanza y aprendizaje y, en consecuencia, favo- recedor del desarrollo humano" (2010, p. 77). Un sistema de educación inclusiva permite transformar el ámbito educativo, proporciona un contexto que fortalece el desarrollo del estudiantado a partir de sus potencialidades, $y$ facilita espacios de respeto y comprensión hacia la variedad de requerimientos de la población estudiantil. Es así, como las estrategias de apoyo conceptualizadas como elemento transformador del contexto educativo que se llevan a cabo en la propuesta curricular, se contemplan como prácticas que responden a las necesidades de aprendizaje del estudiantado con discapacidad, para asegurar la accesibilidad al plan educativo de manera flexible, en todos sus componentes (objetivos, metodología y evaluación). De igual manera, la educación inclusiva promueve acciones que rescatan las habilidades del estudiantado y la eliminación de las barreras de aprendizaje, las cuales se visualizan como los obstáculos que limitan el proceso escolar, por lo cual se requiere que el profesorado, asuma una posición de liderazgo en relación a las estrategias de apoyo, entre otros, para que el estudiantado logre un desarrollo integral, acorde a sus destrezas y capacidades. Asimismo, el uso de estas estrategias ofrece, a la población estudiantil con discapacidad, la oportunidad para alcanzar su máximo potencial en la cotidianidad escolar, en donde la implementación de ese derecho conlleva a la transformación de un sistema educativo más justo y accesible; haciendo realidad una educación de calidad para toda la comunidad estudiantil. 


\section{Requerimientos del estudiantado con dis- capacidad visual en los procesos evaluativos}

La población estudiantil con discapacidad visual al igual que sus pares videntes, deben someterse a los requerimientos y pautas de calidad en el desarrollo del plan escolar, por lo que el profesorado debe determinar los ajustes de manera integral; sin embargo, es en el proceso evaluativo donde la práctica escolar evidencia mayores dificultades para asumir el proceso en condiciones de equidad, considerando las necesidades y destrezas del alumnado. Al respecto, Ibarra, Rodríguez y Gómez (2012), indican que la evaluación del aprendizaje se fundamenta en una concepción abierta y flexible del conocimiento, en donde se hace uso de estrategias que promueven las oportunidades de aprendizaje del estudiantado. En esa misma línea conceptual, el Ministerio de Educación Pública de Costa Rica señala la importancia de:

Considerar la evaluación como un proceso integral, una actividad continua, permanente y flexible que incluye múltiples métodos y operaciones. Orientar la identificación, recopilación, organización y análisis de la información en función de algún propósito específico. Comprobar que la información satisfaga los criterios de validez y confiabilidad, entre otros. Presentar de acuerdo con la información obtenida, alternativas que ofrezcan mayores posibilidades para mejorar la práctica educativa. Elegir entre diferentes opciones de decisión con el fin de responder ade- cuadamente a una situación que requiere ser modificada (2012, p. 3$)$.

La asignación de apoyos de acceso en los procesos evaluativos para la población con discapacidad visual persigue, al igual que para otras poblaciones, que los puntajes de la totalidad de la población examinada sean comparables entre sí y que se puedan hacer interpretaciones legítimas acerca del nivel de cada persona en el atributo que se desea medir (Messick, 1995; Cox, Herner, Demczyk y Nieberding, 2006). Tobin y Hill (2011) indican que para evaluar al estudiantado con discapacidad visual no se puede hacer uso de las prácticas convencionales de los procesos de evaluación, ya que la mayoría están constituidos con imágenes y referencias visuales. Así, existen especificaciones que deben guiar la selección de adecuaciones para esta población; por ejemplo, Suárez y Castellano (2011) citan la importancia de contar con la información médica y oftalmológica de la persona como insumo para determinar las adecuaciones que recibirá, lo que incluye el diagnóstico específico y la estimación de la agudeza y campo visual, de manera que se den solamente las adecuaciones y apoyos atinentes a sus necesidades.

\section{Apoyos y adecuaciones al estudiantado con discapacidad visual}

La asignación de los apoyos estrictamente dirigidos a las necesidades específicas de cada persona es resaltada también por Smarter Balanced Assessment Consortium (2014), quienes afirman que ciertas adecuaciones pueden tener mayor carga cognitiva o dar 
problemas a quienes se les asigne sin necesitarlas. Esta misma organización enumera, entre las adecuaciones en procesos evaluativos pertinentes para personas con discapacidad visual, la transcripción de las pruebas en braille, la aplicación con apoyo de algún software lector de pantalla, formas de respuesta alternativas, pruebas impresas (en el caso de pruebas regularmente aplicadas en computadora), escribiente, software para transcribir las respuestas dictadas por la persona evaluada, magnificación de textos y material visual, incremento de espacios en blanco, formatos simplificados y subrayado.

Los autores citados también enumeran algunos ajustes que, dependiendo de cómo el docente desarrolla la evaluación, va a condicionar el desempeño del estudiantado con discapacidad visual; y por ende su medición, estas incluyen: añadir material visual a los ítems, partes del texto en negrita (para resaltar ideas o dar "pistas"), lenguaje simplificado, cambiar el orden de los distractores (opciones de respuesta que no son correctas) o "disimular" los más atractivos, fragmentación del texto, reordenamiento de ítems, proporcionar dentro del texto preguntas orientadoras o cajas de sugerencias.

Las recomendaciones citadas en los párrafos previos, si bien son importantes para garantizar la accesibilidad de pruebas a personas con discapacidad visual, no omiten la necesidad de que desde el momento del desarrollo de un proceso evaluativo se apliquen ciertos criterios para garantizar su accesibilidad. Estos criterios incluyen la capacitación de las personas constructoras de ítems en lo referente al diseño universal del aprendizaje y los principios de accesibilidad, el desarrollo de ítems accesibles, la delimitación de los objetivos y el contenido de la evaluación, así como la participación en la investigación sobre los efectos de los cambios individuales y combinaciones de cambios destinados a aumentar el diseño universal y la accesibilidad (Smarter Balanced Assessment Consortium, 2014).

Por su parte, Allman (2009) incluye, como parte de una guía para la accesibilidad de pruebas aplicadas a la población con discapacidad visual, las siguientes recomendaciones para elaborar ítems accesibles en el proceso de evaluación:

1. Inclusión de educadores con especialización en el área de discapacidad visual en el proceso de desarrollo de los ítems.

2. Revisión de todos los ítems por parte de personas familiarizadas con el tema de la discapacidad visual para asegurar que ninguno presente sesgo o sea discriminatorio hacia esta población.

3. Incluir tanta información para la resolución del ítem como sea posible en el texto de estos, de manera que se evite la introducción de imágenes que impidan la transcripción del ítem a braille, letra ampliada audio o lector.

4. En general, mantener el lenguaje específico a situaciones visuales, por ejemplo: “observe la siguiente lista..." 
5. El banco de ítems disponible debe ser suficientemente grande para poder reemplazar ítems eliminados, debido a sesgo o la imposibilidad de adaptarlos a formatos accesibles.

6. En caso de que vaya a hacerse una pre experimentación de los ítems, debe incluirse en la muestra una cantidad representativa de personas con discapacidad visual.

7. Todos los materiales de práctica deben ser proveídos en formatos accesibles.

8. Se deben tomar las previsiones para realizar análisis para ítems en formato accesible.

\section{Pautas y criterios en la asignación de los apoyos en el proceso de evaluación}

En lo referente a investigaciones realizadas sobre la utilidad de seguir pautas en los apoyos para la población con discapacidad visual, es posible encontrar algunas referidas a las adecuaciones de lector (humano o software), uso de braille o eliminación de ítems con contenido visual. Ejemplos de estas investigaciones se reseñan en los párrafos siguientes:

Una adecuación muy utilizada para hacer accesibles las evaluaciones a personas con discapacidad visual es la lectura en voz alta, por parte de un lector, de cada uno de los ítems. Respecto a esta adecuación Kim (2012) encontró que los estudiantes con discapacidad visual se desempeñan mejor en pruebas cuando se les proporciona esta adecuación que sin ella; por ejemplo, aplicando la prueba solamente con formatos adaptados (braille, letra ampliada), minimizar la necesidad de realizar una lectura repetida del texto o solamente con tiempo adicional. De acuerdo con sus resultados, el rendimiento académico de los estudiantes con discapacidad visual podría ser medido de forma más justa y precisa mediante el apoyo de un lector, sin que esto les proporcione una ventaja injusta sobre las personas sin discapacidades que aplican la misma prueba.

Otra modificación que suele considerarse para la adaptación de pruebas para población con discapacidad visual es la eliminación de ítems con contenido visual. Esta adecuación debe manejarse con cuidado ya que, dependiendo del constructo medido mediante la prueba o escala, la eliminación de ítems puede llevar a la reducción de la sensibilidad de este para medir ciertas dimensiones del constructo (Wittich, Phillips, Nasreddine y Chertkow, 2010). Si se decide recurrir a esta adecuación, se debe analizar su impacto en la medición y cómo esto afecta los objetivos perseguidos mediante la aplicación de la prueba y, de ser posible, buscar formas alternativas de medir las dimensiones omitidas para no afectar la validez en las mediciones.

La lectura en voz alta de una prueba también se puede realizar mediante la utilización de softwares de lectura de pantalla. Este método ha resultado ser bastante efectivo para la adaptación de test, siempre y cuando los estudiantes tengan la posibilidad de configurar el software (velocidad de lectura, tipo de voz, lectura de signos de puntuación; entre otros), 
de acuerdo con sus preferencias y necesidades (Beal,Rosenblum y Smith, 2011).

El uso del braille también es un recurso útil que no se considera como amenaza a la validez y confiabilidad de las pruebas. $\mathrm{Al}$ respecto, se ha encontrado que la transcripción de ítems a braille no lleva a diferencias significativas en el desempeño de lectores adultos de este sistema; y las personas sin discapacidad visual que aplican la versión impresa de una misma escala (Posey y Henderson, 2012). Por otra parte, existen adecuaciones que no se consideran aptas para garantizar la accesibilidad de personas con discapacidad visual a evaluaciones a gran escala. Entre estas se encuentra la adaptación de gráficos e imágenes mediante recursos táctiles o impresión en relieve, ya que para su utilización debe garantizarse el entrenamiento y práctica suficiente por parte de las personas evaluadas durante su formación académica previa, así como la calidad de la adaptación y de los materiales utilizados (Zebehazy y Wilton, 2014).

Con respecto a la resolución de contenidos matemáticos, se rescata que en esta área no se suele evidenciar situaciones de mayor complejidad que las que determina el requerimiento específico del estudiantado para hacer uso de materiales y recursos adaptados, como el uso del ábaco, la calculadora parlante e instrumentos aritméticos en relieve. De igual forma, se sugiere prescindir de ítems con presencia de operaciones extensas, largas secuencias de datos y excesivo detalle entre el enunciado a resolver y opciones de respuesta
(Peralta y Narbona, 2002). Así, se debe considerar como prioridad proporcionar al grupo de estudiantes las adaptaciones necesarias, por lo cual es relevante que se preste especial atención en los elementos conceptuales de la enseñanza (contenidos, evaluación, materiales) (Cox y Dykes, 2001).

Desimone y Carlson (2004) señalan que un aspecto que asegura la validez de las interpretaciones derivadas de los puntajes obtenidos en un instrumento, es que la persona que lo responde comprenda las preguntas que se plantean de manera similar a quienes diseñaron el instrumento; con lo cual hay que evitar el uso de ítems de extensión larga y con mucho detalle, ya que el contraste excesivo de referencias, datos o elementos diversos, hacen complejo el proceso secuencial de la lectura.

Por ejemplo, alusiones a descripciones y vocabulario con componentes visuales para su resolución, poniendo en posición de desventaja al estudiantado con discapacidad visual; ya que al recibir las imágenes en forma distorsionada (por la baja visión) o no recibirla (por su condición de ceguera), se les dificulta establecer las relaciones o analogías para replantear y organizar la información. Por otra parte, al realizar la lectura de textos, un campo visual limitado (causado por deficiencias del ojo o por las dificultades de enfoque a distancia) restringe el uso de la información que se obtiene en la periferia del texto, explicando un proceso de lectura más lento en el estudiantado con baja visión (Gompel, Van Bon y Schreuder, 2004). En cuanto al grupo de estu- 
diantes ciegos usuarios del sistema Braille, se reconoce que realizan una percepción global de la palabra y la frase desde un proceso analítico, por lo que la lectura y la comprensión se ve afectada por la incidencia de errores de exactitud, como son añadir u omitir puntos que inciden en la precisión de la información recibida (Peralta y Narbona, 2002); por lo cual, además, es conveniente el uso y la disposición de mayor tiempo para llevar a cabo el proceso lector en condiciones de equidad.

\section{Conclusiones}

El impacto educativo que conlleva la elaboración de una propuesta curricular con todos sus componentes (contenidos, actividades y proceso evaluativo), considerando los requerimientos de la población estudiantil con discapacidad visual, comprende prácticas relacionadas con las estrategias de flexibilización de los mismos, para dar respuesta efectiva a la diversidad de requerimientos del estudiantado en procura de condiciones de equidad, en el acceso al proceso de enseñanza aprendizaje.

En ese sentido, los retos de la educación actual se debaten entre una realidad compleja, en donde persisten las barreras de aprendizaje y los esfuerzos por contribuir e incidir en los nuevos desafíos; y lograr un sistema educativo inclusivo, que favorezca procesos de formación accesibles, garantizando una participación efectiva de la población con discapacidad visual.

El proceso de flexibilización en los procesos evaluativos debe generarse en corresponden- cia con los apoyos de acceso al contenido de los mismos, no así al nivel de dificultad de las habilidades o asignaturas a valorar, esto en función de la búsqueda de un proceso de enseñanza aprendizaje que elimine la situación de desventaja y desigualdad a los que se enfrentan el estudiantado con discapacidad visual. Es así, como esta situación genera acciones que favorezcan entornos accesibles, en los cuales prevalezcan apoyos en los diferentes espacios de comunicación e información, en una relación de interdependencia con la población estudiantil.

Los ajustes y modificaciones que se realizan para llevar a cabo procesos evaluativos adecuados a los requerimientos del grupo de estudiantes con discapacidad visual, suponen el ejercicio de una flexibilización de la forma en que se presentan y se relacionan con la adaptación a diversos formatos (tamaño, grosor, tipo de letra, audible, digital); así como al uso del tiempo y de apoyos técnicos. Uno de los retos más significativos, son los criterios en la estructura y presentación, en procura de procesos evaluativos comprensibles y accesibles, como un componente clave para la plena participación del estudiantado hacia el ejercicio del derecho a una educación de calidad.

Tener claridad de las necesidades y particularidades del estudiantado con discapacidad visual, permite a la comunidad educativa - y en particular al profesorado - potenciar el desarrollo de la población estudiantil y eliminar las barreras que limitan el proceso de enseñanza aprendizaje; de ahí la importancia de 
priorizar acciones que dinamicen la propuesta curricular, en coherencia con una postura de defensa al derecho a la educación de calidad.

El entramado de concepciones y estrategias de apoyo en la propuesta educativa, que se da desde el modelo de educación inclusiva, debe generar espacios en donde tanto la población con discapacidad como el sistema educativo, coincidan con propuestas y toma de decisio- nes en conjunto, con el fin de contribuir a la mejora de los procesos curriculares. A partir de esto, es necesario continuar profundizando en la temática a partir de futuras investigaciones, tomando en cuenta las prácticas que se realizan desde las políticas y acciones nacionales e internacionales, para favorecer la reflexión, el análisis y el posicionamiento de sistemas educativos inclusivos.

\section{Referencias}

Aquino, S.; García, V. e Izquierdo, J. (2012). La inclusión educativa de ciegos y baja visión en el nivel superior. Un estudio de caso. Revista Electrónica Sinéctica, número 39, Instituto Tecnológico y de Estudios Superiores de Occidente; México. Recuperado de http://www. sinectica.iteso.mx/index.php?cur=39\&art=39_12

Allman, C. (2009). Test Access: Making tests accessible for students with visual impairments. (Cuarta edición) (Acceso a Pruebas Accesibles para Estudiantes con Discapacidad Visual. Louisville, Kentucky: American Printing House for the Blind. Recuperado de http://www. aph.org/files/tests/access2/access2.pdf

Beal, C.R.; Rosenblum L.P. y Smith, D.W. (2011). A Pilot Study of a Self-voicing Computer Program for Prealgebra Math Problems (Un estudio piloto de programas de auto voz para problemas matemáticos de prealgebra). Journal of Visual Impairmente Blindness, 105 (3), 157-169

Blanco, R. (2010). Sistema Regional de Información Educativa de los Estudiantes con Discapacidad (SIRIED). Propuesta Metodológica. Santiago de Chile. UNESCO. Recuperado de http://www.unesco.org/new/fileadmin/MULTIMEDIA/FIELD/Santiago/pdf/SIRIED

Brown, F. (1980). Principios de la mediación en psicología y educación. México, D.F.: Editorial El Manual Moderno, S.A. de C.V.

Costa Rica, Ministerio de Educación Pública. (2012). Dirección de Desarrollo Curricular. Departamento de Evaluación de los Aprendizajes. La Evaluación de los aprendizajes en el contexto de la atención de las necesidades educativas de los estudiantes. Ministerio de Educación Pública. - 2. Ed.- San José: Cóndor Editores. 
Cox, M.L.; Herner, J.G.; Demczyk, M.J. y Nieberding, J.J. (2006). Provision of testing accommodations for students with disabilities on statewide assessments: Statistical links with participation and disciplines rates. Remedial and Special Education, 27(6), 346-354

Cox, P.R. y Dykes, M.K. (2001). Effective classroom adaptations for students with visual impairments. Teaching Exceptional Children, 33(6), 68-76

Desimone, L. M., y Carlson, K. (2004). Are we asking the right questions? Using cognitive interviews to improve surveys in education research. Educational Evaluation and Policy Analysis, 26 (1)

Gompel, M.; Van Bon, W. y Schreuder, R. (2004). Word read and processing of the identity and order of letters by children with low vision and sighted children. Journal of Visual Impairment \& Blindnes, 757-772

Guirao-Goris,J.A.; Olmedo Salas, A.; Ferrer Ferrandis, E. (2008) El artículo de revisión. Revista Iberoamericana de Enfermeria Comunitaria, 1, 1, 6. Recuperado de http://revista.enfermeriacomunitaria.org/articuloCompleto.php? $\mathrm{ID}=7$

Ibarra, M. S.; Rodríguez, G. y Gómez, M. Á. (2012). La evaluación entre iguales: beneficios y estrategias para su práctica en la universidad. Revista de Educación, 359. Septiembrediciembre 2012, pp. 206-231. Recuperado de https://www.researchgate.net/publication/286211647_La_evaluacion_entre_iguales_Beneficios_y_estrategias_para_su_practica_en_la_universidad

Instituto Nacional de Estadística y Censos. (2011). X Censo Nacional de Población y VI de Vivienda. Recuperado de https://www.cipacdh.org/pdf/Resultados_Generales_Censo_2011.pdf

Kim, J. S. (2012). The Effect of "Read-Aloud" as a test accommodation for students with visual impairments in South Korea. Journal of Visual ImpairmentઐBlindness, 106 (6), 356-361

León, B.; Polanco, B.; Rovezzi, G.; Contreras, N. y Ramos, P. (2014). El equipo multidisciplinario en la atención de alumnos con baja visión como una práctica educativa para la inclusión. Consejo Internacional para la Educación de Personas con Discapacidad Visual (ICEVI). Recuperado de:http://icevi.org/pdf/Baja_Vision_(Low_Vision_book_in_Spanish).pdf

López, M. (2004). Aspectos evolutivos y educativos de la deficiencia visual. Primera Edición. España: Editorial Netbiblo

Messick, S. (1995). Validity of psychological assessment: Validation of inferences from person's responses and performances as scientific inquiry into score meaning. American Psychologist, 50, 741-749 
Parra, C. (2010). Educación inclusiva: un modelo de educación para todos. Revista ISEES, No.8, pp. 73-84. Recuperado de: Dialnet-EducacionInclusivaUnModeloDeEducacionParaTodos-3777544.pdf

Peralta F. y Narbona J. (2002). Deficiencia visual en el niño. Estudios sobre educación, No.02, pp. 35-52. ISSN 1578-700. Universidad de Navarra. Recuperado de: http://hdl.handle. net/10171/7998

Posey, V.K. y Henderson, B.W. (2012). Comprehensive Adult Student Assessment Systems Braille Reading Assessment: An Exploratory Study. Journal of Visual Impairment \& Blindness, $106(8), 488-499$

Reed, M. y Curtis, K. (2012) Experiences of Students with Visual Impairments in Canadian Higher Education. Journal of Visual Impairment \& Blindness, 106, 7, pp. 414-425. Smarter Balanced Assessment Consortium (2014). Usability, accessibility, and accommodations Guidelines. Recuperado de http://www.smarterbalanced.org/smarterbalanced-assessments/

Suárez, J.M., y Castellano, J.R. (2011). Atención educativa a personas ciega y con baja visión en la Sierra de Cádiz. Clave XXI Reflexiones y Experiencias en Educación 6, 1 - 16

Tauber, F. (2011). La inclusión en la universidad pública argentina. En Katz, S., y Mara, P. (compiladoras). Hacia una universidad accesible: construcciones colectivas por la discapacidad. Buenos Aires, Argentina: Editorial de la Universidad Nacional de la Plata

Tobin, M. y Hill E. (2011). Issues in the educational psychological assessment of Visually impaired children: Test-retest reliability of the Williams Intelligence Test for Children with Defective Vision. Revista The British Journal of Visual Impairment, 29(3), pp. 208-214.

Wittich, W.; Phillips, N.; Nasreddine, Z. S. y Chertkow, H. (2010). Sensitivity and specificity of the Montreal Cognitive Assessment modified for individuals who are visually impaired. Journal of Visual Impairment \& Blindness, 104, 360-368

Wyver Shirley, R. y Markham, R. (1999). Divergent thinking of children with severe visual impairments. Journal of Visual Impairment \& Blindness. 93 (4), 233

Zebehazy, K.T., y Wilton, A.P. (2014). Quality, importance, and instruction: The perspectives of teachers of students with visual impairments on graphics use by students. Journal of Visual Impairment\&Blindness, 108(1), 5-16. 